\title{
Neuromuscular Fatigue in Contact Sports: Theories and Reality of a High Performance Environment
}

\section{Pierre Austruy}

Department of Sports, Victoria University, Australia

*Corresponding author: Pierre Austruy, Department of Sports, Victoria University, Australia, Tel: +61 3 9919 6100; E-mail: PierreA@sydneyroosters.com.au

Received date: July 29, 2015; Accepted date: September 24, 2016; Published date: September 30, 2016

Copyright: (c) 2016 Austruy P. This is an open-access article distributed under the terms of the Creative Commons Attribution License, which permits unrestricted use, distribution and reproduction in any medium, provided the original author and source are credited.

Keywords: Neuromuscular fatigue; Performance enhancement; Sports; Voluntary contraction; CNS

\section{Introduction}

Evolving in a highly competitive environment where maximal performance is all that matter, elite athletes are required to allocate training time to an intensity that mimics the sport-specific characteristics they experience during competition [1]. Due to the demands of training and competition, athletes experience a constant cycle of "fatigue-recovery-adaptation". Performance is enhanced by training stimulus only if adaptive processes are greater than the induced fatigue, and as such, the monitoring of fatigue is important to determine appropriate training loads to maximize subsequent performance [2]. Historically, fatigue has been defined in accordance with the varied sub disciplines associated with sports science, namely the divisions of physiology, psychology and biomechanics. At one extreme, Physiologists may consider fatigue as failure or dysfunction of a particular physiological system [3] while at the other extreme, psychologist may view fatigue as an uncomfortable perception or sensation [4].

To describe neuromuscular fatigue, a biomechanical approach appears to be the most appropriate. Neuromuscular fatigue is traditionally associated with changes anywhere on the pathway between the brain and muscle fibre, with effects at the motor unit level considered peripheral and events occurring in the brain or spinal cord considered central [5]. The level of force produced by a muscle in a voluntary contraction is determined by the firing frequency and pattern of motor unit recruitment, and increasing either of these aspects plays a role in increasing the force of the contraction [6]. Fatigued muscle is known to develop tension more slowly than nonfatigued muscle and displays a simultaneous extension of the relaxation phase in conjunction with a decrease in peak force production capability [7]. It has also been suggested that this reversible reduction in efficiency may be present regardless of whether or not a given workload can be maintained [8]. The exact site on the chain from the Central Nervous System (CNS) (Central Activation Failure) to the contractile tissue (neuromuscular propagation failure) where neuromuscular fatigue is controlled is unclear; and it has been reported that both central and peripheral factors are responsible for reduced force production capability [9]. Research aimed at determining the site and most appropriate method of measuring neuromuscular fatigue has been pursued quite vigorously. As we understand, monitoring fatigue and especially neuromuscular fatigue is a critical part of any high performance monitoring program. In order to do so, different tools and methods are available for consideration.

\section{How to Monitor it}

\section{Measure of maximal voluntary contraction (MVC)}

Reduced output of motor neurons is thought to occur as a result of the attempt by the CNS to optimize force output under fatigue conditions. Due to this, various EMG measures are considered effective in quantifying neuromuscular fatigue [10]. Numerous studies have investigated neuromuscular fatigue thanks to this technology. In a study utilizing EMG to measure fatigue, Bonato et al. [10] examined fatigue related changes in the EMG signal of trunk and limb musculature during a repetitive lifting task. They calculated an EMG based fatigue index for dynamic contractions utilizing the instantaneous median frequency. This measure showed a significant decrease during a cyclic lifting task $(\mathrm{p}<0.05)$ and mean MVC force recorded immediately after the task was also significantly reduced $(p<0.01)$ when compared to baseline measures. In the same study, maximum torque of working muscles significantly decreased during the lifting task.

In a series of investigations, Sogaard et al. [11] postulated that MU recruitment and the synchronisation of MU firing patterns as well as changes in these features may be revealed via mechanomyogram (MMG). Simultaneous measurements of EMG and MMG have been used to examine the dissociation between the electrical and mechanical components that occur during neuromuscular fatigue. The amplitude of MMG signal has been related to MU recruitment and it has been suggested that the MMG frequency content may provide information about MU firing rate. The amplitude of surface EMG signals however reflects muscle activation whereas during isometric muscle actions EMG is influenced by action potential conduction velocities of the active muscle fibres [11]. If the both EMG and MMG technologies are accurate and reliable measurements tools and present some interests for diagnosis of neuromuscular fatigue, it appears to present some major limitations.

Firstly, in the context of contact sports, Incorporation of EMG and MMG analysis during field testing protocols is problematic and restricted by the non-functional characteristics of the fatigue inducing exercise protocols used in this form of muscle assessment, such as isokinetic leg extensions and isometric elbow flexion movements. Secondly, MVC portray little resemblance to muscle recruitment and activation patterns during a bout of dynamic exercise involving submaximal muscle contractions. Isometric force measures have also been reported to underestimate functional impairment of neuromuscular performance and as such isometric MVC may be a poor measure of dynamic exercise performance [12]. Furthermore, isokinetic muscle contractions may not replicate normal muscle contraction patterns that are characteristic of dynamic sports performance, such as acceleration, deceleration and ballistic running 
and jumping activities. Monitoring neuromuscular fatigue in contact sports in a high performance environment stress the need for more specific testing procedure that are time efficient, easy to set up and that gives easy to interpret data.

\section{Jump tests}

Recent evidence suggests the incorporation of dynamic movements involving the Strength Shortening Cycle provides a more specific examination of neuromuscular fatigue in these settings. Exercise involving the SSC incorporates metabolic, mechanical and neural elements of fatigue together with impairment of the stretch-reflex activation. Typically, the SSC involves a pre-activated muscle that is first stretched (eccentric action) and then shortened (concentric action) and is common to exercise such as running, jumping or hopping [13]. Recovery from SSC exercise occurs in two phases that are characterised by an initial large drop in performance, followed by a transient recovery and then a further drop that generally peaks 48 to $72 \mathrm{~h}$ post exercise [14]. The initial decline in the ability to produce force has been attributed to metabolic processes and the secondary reduction to inflammatory processes.

Various forms of the CMJ and SJ have been used to assess neuromuscular performance. Fundamental differentiations of CMJ and SJ technique is determined via the exclusion or inclusion of arm swing and lower limb countermovement during execution of the eccentric/preparation and concentric/take off phases of the respective jump technique. Jump height, power peak, flight time and RFD during the CMJ have been used extensively to assess sports performance and neuromuscular fatigue [15-17]. And it has been proposed that assessing repetitive $\mathrm{VJ}$ power may also be of interest [15]. It is interesting to note that the elastic behaviour of muscles has been shown to be similar in VJ and running, suggesting VJ may be highly relevant for assessing various parameters important in sport [17].

The functional impairments induced by prolonged, continuous activity, such as running and cycling have been well documented [18-20], however less is known regarding the force and power development characteristics of the neuromuscular system following sports performance involving prolonged, high-intensity intermittent exercise.

In a particular study aimed at assessing the fatigue response to $180 \mathrm{~min}$ of tennis match play, neuromuscular fatigue was assessed with the use of a SQJ, CMJ and $15 \mathrm{~s}$ of repeated CMJ's [21]. Countermovement jump peak power post match was significantly different to pre $(\mathrm{p}<0.05)$ whilst both $\mathrm{SQJ}$ and $\mathrm{CMJ}$ peak power continued to be depressed $30 \mathrm{~min}$ post game compared to pre $(\mathrm{p}<0.001$ and $\mathrm{p}<0.05$ respectively). Interestingly there was no change to leg stiffness as measured via the repeated CMJ test.

Dynamic peak Force (PF), peak rate of force development (PRFD) and peak power (PP) have been identified as key strength and power measures that are fundamental to both VJ and sports performance [22-26]. The VJ has been used extensively since the 1920's as a training and sports specific assessment modality of neuromuscular performance in athletes given the similarity of many sports specific movements and activities to the jumping movement [27,28]. Recently, the VJ has become an important inclusion in talent identification, preparticipation screening and long term athlete development models for the purposes of athlete profiling. Accordingly, the VJ has been found to be a reliable predictor of successful performance in sports such as American Football [29], Rugby League [30-32] and Soccer [33]. In studies [30,31] that have compared lower body strength and power characteristics of professional and college-aged Rugby League players, Baker et al., $[30,31]$ reported significantly $(p<0.05)$ greater measures of $\mathrm{PF}, \mathrm{AP}$ and $\mathrm{PP}$ for professional players during one repetition maximum (1RM) squat and SJ tests. They concluded that dynamic measures of strength and power may be used to discriminate between elite and subelite Rugby League players, with greater strength and power performance characteristics in elite Rugby League players.

For many sports including elite Rugby League match-play, successful performance is coupled with high levels of strength [30-35]. During CMJ performance, not only is there a considerable force and power requirement, but force must be exerted at a rapid rate to facilitate optimal sports performance [36]. The RFD is an index of explosive strength and reflects the capacity to exert maximal force in minimal time [37]. The RFD has important functional significance in rapid and forceful skeletal muscle contractions during activities such as sprinting, throwing and jumping, which typically involve muscle contraction times of $<250 \mathrm{~ms}$ [38-40]. In contrast, the time to reach maximum force in most human skeletal muscle is $>250 \mathrm{~ms}$ [41]. Therefore, during rapid acceleration, deceleration or change of direction movements that are characteristic of sports performance, short skeletal muscle contraction times may not permit maximal force to be reached. As a result, the early increase in RFD becomes important to produce a higher level of force during the initial 100-200 ms of muscle contraction [38]. The RFD may be considered to be more specific than other force-time measures in athletes because in most sports, the time available to produce force is limited to $<250 \mathrm{~ms}$ [42]. Although RFD has been shown to be an important performance variable [42-44], with athlete development models such as that outlined by Siff [46] indicating that the expression of explosive strength as a key element in athlete progression to elite levels.

If Vertical jump does offer reliable and accurate measures of neuromuscular fatigue in contact sports, and if various variable can be investigated with some strongly linked to athletic performance (i.e RFD), that unfortunately doesn't come without limitations. The evidence regarding the ability of SSC tasks such as the CMJ to detect neuromuscular fatigue and performance decrement following team sport match-play is limited, and those data that do exist are conflicting [44-46]. It is comprehensible that contact sports such as Rugby league or Football are involving a high amount of high intensity intermittent efforts, frequent maximal and sub-maximal SSC activity, as well as bruising and traumas induced by collisions. All the above mentioned are expected to cause considerable acute metabolic/peripheral fatigue in conjunction with prolonged central fatigue during the post-match recovery period. In the reality of a high performance environment, testing neuromuscular fatigue on a weekly (daily) basis through an energetically demanding test such as vertical jump is uneasy. Postgame, metabolic fatigue, inflammation, injuries, bruises and knocks plus a lack of motivation can greatly violate the testing procedure accuracy. If it seems almost perfect in pre-season, jumps testing appears to be difficult to set up during the competitive season. Therefore, tests that are less demanding energetically and less susceptible to be influenced by metabolic residuals or injuries would be more appropriate for contact sports.

\section{Grip strength}

It is important to admit that in the context of high performance environment and especially team sports, accuracy is sometimes secondary to reliability and feasibility. Expensive and voluminous 
laboratory equipment are both accurate and reliable but inconvenient and too time consuming for weekly assessments of neuromuscular fatigue in contact sports. Jump tests despite supported by a solid literature are confronted to important physiological and motivational limitations in the context of a high performance monitoring programme. Therefore, the objective is to find a test giving a single main data, accurate enough but easy to set up, control, interpret and report. As most team sports are competing in championships involving frequent travels, neuromuscular fatigue testing should involve a minimal and transportable material. Some interesting tools available on the market offers to test similar variables as the $\mathrm{Vj}$ ones but using more specialized movements (i.e., Gym aware, bar sensei etc.). However, despite being of decent accuracy and easy to use and transport, these accelerometers don't meet the last fundamental criteria: neuromuscular testing should not be altered by peripheral fatigue.

The handgrip test has gain interest in high performance programmes to monitor neuromuscular fatigue due to its potential for meeting all the criteria of a perfect test (inexpensive, transportable, user friendly, doesn't require movements). Unfortunately, the poor accuracy makes this test inappropriate. Recently, some interesting research was presented at the International Colloquium on Sports Science, Exercise, Engineering and Technology that investigated the relationship between hand grip strength and total body strength and power measures. A total of 100 high level male athletes approximately 20 years of age from various sports were included in this study. One rep $\max (1 \mathrm{RM})$ values were acquired in the lat pull down, incline bench press, leg press and leg extension. Hand grip strength was assessed via hand dynamometer and vertical jump values were assessed with a Vertec device. Interestingly, the hand grip strength showed no significant correlation with any of the 1RM values. Less surprisingly, hand grip strength showed virtually no correlation with vertical jump values. The author's concluded that hand grip strength was not a very accurate measure or predictor of total body strength or power in high level athletes.

\section{Is proprioception the answer}

First, what are proprioceptors? Proprioceptors are special sensory nerves located in muscles, joints and tendons of the body. They have a very special role in recording data from the musculoskeletal system such as when muscles tissues are shortened, compressed, twisted, etc., and feed that data to the brain so that the brain knows what the body is doing and where it's parts are in space.

Thankfully, proprioception is auto-regulated by the body and does not necessarily requires conscious thought on our part; which brings to the first point about proprioception and its importance in athletics. Basically, training through repetitions the body to perform movements can result in a certain automatism (often referred as muscle memory). Actually training is stimulating the feedback loop between the proprioceptors and the cerebellum, the part of the brain that controls posture, balance, and smooth muscle movement. This entire process can be completely autonomous and does not necessarily require conscious effort on our part. If the loop is working well, if the CNS is fresh and the movement have been practiced a lot, then if we try and think our way through this athletic or dynamic movement, we get less than satisfactory results. The message is, once you've learned a specific motor pattern, be it an Olympic lift, bench press, or javelin throw, get out of your own way and let your body do 'auto-magically' what you've taught it to do; by doing so you will be more efficient and precise.
Another role of proprioception worth being discussed concerns power athletes, i.e., power lifters and athletes competing in contacts sports such as footballers. There is one particular type of proprioceptive nerve that is involved with regulating and preventing damage to muscles and tendons. For those athletes that have reached the elite levels, they have produced enough strength in their muscles to literally tear the muscle tendon from the bone, and in rare cases, even break the bone to which the tendon attaches. The golgi tendon organ (or GTO) is the proprioceptor nerve that is responsible for preventing damage to the muscles and tendons. The golgi tendon organ is a multibranched sensory nerve ending located in the tendons that attach the muscles to the bones. This incredible nerve actually measures the tension in muscle and monitors the amount of force pulling on the bone where the tendons attach. Of course like anything this process isn't always reliable, especially with novice lifters. The main role of the GTO is to prevent ripping of connective tissue or muscles. In the case of experienced lifters or pro athletes, especially those with a lot of training time and are at elite performance levels, they have actually learned to bypass or override the GTO response which is obviously potentially dangerous. Elite level lifters are constantly walking a fine line between maximum performance and potentially career ending injuries.

All of that is to say that the potentially most dangerous aspect of CNS fatigue isn't necessarily a loss of power output or a diminution of muscles innervation. CNS fatigue does affect directly proprioceptive abilities, which may be the most critical ones to prevent injury and perform well. And admitting it raises an important question. Is testing proprioceptive ability is of an upmost importance to prevent injuries and monitor neuromuscular fatigue in contact sports? Why not testing neuromuscular fatigue by testing proprioceptive ability in contact sports?

Simple test for CNS could then be to ask your athletes to perform 10 consecutives reps of the most basic specific movement to their speciality (10 serves for a tennis player, 10 shots for a basketball player and so on) and to record accuracy, quality and efficiency of movement. These data could help tracking when CNS fatigue is a concern and when readiness is optimal. Specific screening that requires balance and focus could also serves as a potential CNS fatigue testing procedure.

\section{Conclusion}

There is a substantial body of work that has attempted to describe the mechanisms and relative contributions of central and peripheral factors to neuromuscular fatigue in contact sports. Researches have utilized EMG, MMG and functional movements such as a CMJ to assess the impact of athletic performance on neuromuscular status. Despite this, little work has investigated the impact of elite level team sport on a wide range of reliable CMJ jump variables in an attempt to determine their expected pattern of response or their suitability for both acute and longer term monitoring of neuromuscular fatigue. The high intensity, intermittent nature of contact sports match-play that includes frequent maximal and sub-maximal SSC activity and blunt force trauma is expected to cause considerable acute metabolic/ peripheral fatigue in conjunction with prolonged central fatigue during the post-match recovery period. Accordingly, analysis of neuromuscular fatigue in contact sports players represents an interesting and important strategy to determine subsequent training loads, quantify decrement in the ability of players to tolerate training and establish the time course of recovery following match-play. High performance programmes offers a challenging environment to conduct 
testing procedures, where time efficiency, reliability, convenience and easiness of data interpretations are of the upmost importance. Focusing on the proprioceptive abilities appears as an innovative approach to neuromuscular fatigue monitoring and should be the object of further researches.

\section{References}

1. Gabbett TJ (2005) Science of rugby league football: A Review. J Sports Sci 23: 961-976.

2. Fowles JR (2006) Technical issues in quantifying low-frequency fatigue in athletes. Int J Sports Physiol and Perf 1: 169-171.

3. Sahlin K (1992) Metabolic factors in fatigue. Sports Med 13: 99-107.

4. Kayser B (2003) Exercise starts and ends in the brain. Eur J Appl Physiol 90: 411-419.

5. Weir JP, Beck TW, Cramer JT, Housh TJ (2005) Is fatigue all in your head? A critical review of the central governor model. Br J Sports Med 40: 573-586.

6. Tarata MT (2003) Mechanomyogrphy versus Electromyography, in monitoring the muscular fatigue. BioMedical Engineering Online.

7. Giannesini B, Cozzone PJ, Bendaham D (2003) Non-invasive investigations of muscular fatigue: metabolic and electromyographic components. Biochimie 85: 873-883.

8. Verin E, Ross E, Demoule A, Hopkinson N, Nickol A, et al. (2004) Effects of exhaustive incremental treadmill exercise on diaphragm and quadriceps motor potentials evoked by transcranial magnetic stimulation. J Appl Physiol 96:253-259.

9. Lepers R, Haussworth C, Maffiuletti N, Brisswalter J, Van Hoecke J (2000) Evidence of neuromuscular fatigue after prolonged cycling exercise. Med Sci Sports Exerc. 32:1880-1886.

10. Bosco C, Luhtanen P, Komi PV (1983) A Simple Method for Measurements of Mechanical Power in Jumping. Eur J Appl Physiol 50: 273-282.

11. Sogaard K, Blangsted AK, Jorgensen LV, Madeleine P, Sjogaard G (2003) Evidence of long term muscle fatigue following prolonged intermittent contractions based on mechano- and electromyograms. J Electromyogr Kinesiol 13: 441-450.

12. Cairns SP, Knicker AJ, Martin W, Sjogaard G (2005) Evaluation of Models Used to Study Neuromuscular Fatigue. Exerc Sport Sci Rev 33: 9-16.

13. Meeusen R, Piacentini MF, Busschaert B, Buyse L, De Schutter G, et al. (2004) Hormonal responses in athletes: the use of a two bout exercise protocol to detect subtle differences in (over)training status. Eur J Appl Physiol 91: 140-146.

14. Komi PV (2000) Stretch-shortening cycle: a powerful model to study normal and fatigued muscle. J Biomech 33: 1197-1206.

15. Howell AK, Gaughan JP, Cairns MA, Faigenbaum AD, Libonati JR (2001) The Effect of Muscle Hypoperfusion-Hyperemia on Repetitive Vertical Jump Performance. J Strength Cond Res 15: 446-449.

16. Bosco C, Ito A, Komi PV, Luhtanen P, Prahkila P, Rusko H, Viitasalo JT (1982) Neuromuscular function and mechanical efficiency of human leg extensor muscles during jumping exercises. Acta Physiol Scand 114: 543-550.

17. Bosco C, Montanari G, Ribacchi R, Giovenali P, Latteri F, et al. (1987) Relationship between efficiency of muscular work during jumping and the energetics of running. Eur J Appl Physiol 56: 138-143.

18. Finni T, Kyrolainen H, Avela J, Komi PV (2003) Maximal but not submaximal performance is reduced by constant-speed $10-\mathrm{km}$ run. J Sports Med Phys Fitness 43: 411-417.

19. Gomez AL, Radzwich RJ, Denegar CR, Volek JS, Rubin MR, et al. (2002) The Effects of a 10-Kilometer Run on Muscle Strength and Power J Strength Cond Res 16: 184-191.

20. Paavolainen L, Hakkinen K, Nummela A, Rusko H (1994) Neuromuscular characteristics and fatigue in endurance and sprint athletes during a new anaerobic power test. Eur J Appl Physiol Occup Physiol 69: 119-126.
21. Girard O, Lattier G, Micallef JP, Millet GP (2006) Changes in exercise characteristics, maximal voluntary contraction, and explosive strength during prolonged tennis playing. Br J Sports Med 40: 521-526.

22. Kraska JM, Ramsey MW, Haff GG, Fethke N, Sands WA, et al. (2009) Relationship between strength characteristics and unweighted and weighted vertical jump height. Int J Sports Physiol and Perf 4: 461-473.

23. Peterson MD, Alvar BA, Rhea MR (2006) The contribution of maximal force production to explosive movement among young collegiate athletes. J Strength Cond Res 20: 867-873.

24. Sleivert G, Taingahue M (2004) The relationship between maximal jumpsquat power and sprint acceleration in athletes. Eur J Appl Physiol 91: 46-52.

25. Stone M, O'Bryant H, McCoy L, Coglianese R, Lehmkuhl M, et al. (2003) Power and maximum strength relationships during performance of dynamic and static weighted jumps. J Strength Cond Res 17: 140-147.

26. Stone MH, Sands WA, Pierce KC, Carlock J, Cardinale M, et al. (2005) Relationship of maximum strength to weightlifting performance. Med Sci Sports Exerc 37: 1037-1043.

27. Harman EA, Rosenstein MT, Frykman PN, Rosenstein RM, Kraemer WJ (1991) Estimation of human power output from vertical jump. J Strength Cond Res 5:116-120.

28. Sargent DA (1921) The physical test of a man. Am Phys Educ Rev 26: 188-194.

29. McGee KJ, Burkett LN (2003) The National Football League combine: a reliable predictor of draft status? J Strength Cond Res 17: 6-11.

30. Baker D (2001) Comparison of upper-body strength and power between professional and college aged rugby league players. J Strength Cond Res 15: 30-35.

31. Baker D (2002) Differences in strength and power among junior-high, senior-high, college-aged, and elite professional rugby league players. J Strength Cond Res 16: 581-585.

32. Baker DG, Newton RU (2008) Comparison of lower body strength, power, acceleration, speed, agility, and sprint momentum to describe and compare playing rank among professional rugby league players. J Strength Cond Res 22: 153-158.

33. Wisloff U, Castanga C, Helgerud J, Jones J, Hoff J (2004) Strong correlation of maximal squat strength with sprint performance and vertical jump height in elite soccer players. Br J Sport Med 38: 285-288.

34. Cronin JB, Hansen KT (2005) Strength and power predictors of sports speed. J Strength Cond Res 19: 349-357.

35. Stone MH, Sanborn K, O'Bryant HS, Hartman M, Stone ME, et al. (2003) Maximum strength-power-performance relationships in collegiate throwers. J Strength Cond Res 17: 739-745.

36. McBride JM, McBride TT, Davie A, Newton RU (1999) A comparison of strength and power characteristics between power lifters, Olympic lifters, and sprinters. J Strength Cond Res 13: 58-66.

37. Zatsiorsky VM (2006) Science and practice of strength training. Champaign Ill: Human Kinetics 251pp.

38. Aagaard P, Simonsen EB, Andersen JL, Magnusson P, Dyhre-Poulsen P (2002) Increased rate of force development and neural drive of human skeletal muscle following resistance training. J Appl Physiol 93: 1318-1326.

39. Newton RU and Kraemer WJ (1994) Developing explosive muscular power: implications for a mixed methods training strategy. Strength and Conditioning 16: 20-31.

40. Wilson G, Lyttle A, Ostrowski K, Murphy A (1995) Assessing dynamic performance: A comparison of rate of force development tests. J Strength Cond Res 9: 176-181.

41. Hakkinen K, Komi PV, Alen M (1985) Effect of explosive type strength training on isometric force- and relaxation-time, electromyographic and muscle fibre characteristics of leg extensor muscles. Acta Physiol Scand 125: 587- 600 .

42. Cronin J, Sleivert G (2005) Challenges in understanding the influence of maximal power training on improving athletic performance. Sports Med 35: 213-234. 
Citation: Austruy P (2016) Neuromuscular Fatigue in Contact Sports: Theories and Reality of a High Performance Environment. J Sports Med Doping Stud 6: 184. doi:10.4172/2161-0673.1000185

Page 5 of 5

43. Siff MC (2000) Supertraining. Denver: Supertraining Institute.

44. Andersson H, Raastad T, Nilsson J, Paulsen G, Garthe I, et al. (2008) Neuromuscular fatigue and recovery in elite female soccer: Effects of active recovery. Med Sci Sport Exerc 40: 372-380.

45. Cormack SJ, Newton RU, McGuigan MR (2008) Neuromuscular and endocrine responses of elite players to an Australian rules football match. Int J Sports Physiol and Perf 3: 359-374.
46. Hoffman JR, Maresh CM, Newton RU, Rubin MR, French DN, et al. (2002) Performance, biochemical, and endocrine changes during a competitive football game. Med Sci Sports Exerc 34: 1845-1853. 